\title{
Resumen de tesis: “Policía y construcción estatal Higiene urbana, vigilancia y territorialidad en Buenos Aires y sus alrededores (1782-1824)"1
}

\author{
María Agustina Vaccaroni \\ (Universidad Nacional de Mar del Plata) \\ magustinavaccaroni@gmail.com
}

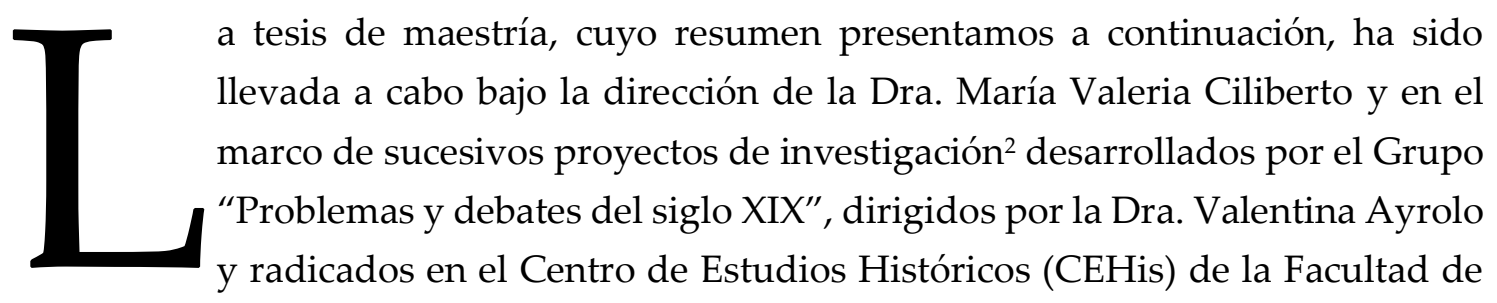
Humanidades de la Universidad Nacional de Mar del Plata. Continúa la investigación iniciada en el marco de la Tesina de Licenciatura, ${ }^{3}$ pero ampliando problemas y objetivos en términos temporales e indagando sobre nuevos ejes y aristas en torno al objeto de estudio, que atienden a la problemática de los primeros indicios de institucionalización de un dispositivo de control policial en la ciudad de Buenos Aires y sus alrededores desde fines del siglo XVIII hasta las primeras décadas del XIX.

El contexto político, social pero también historiográfico de esta tesis es uno en el cual la policía y su accionar, en muchos casos brutal, ha sido puesto en el ojo público. Este cuadro de situación es el trasfondo de la renovación historiográfica que fue preludiada por ciencias sociales como la antropología, la sociología y las ciencias políticas, pero que en las últimas dos décadas ha alcanzado a la historia, permitiendo la puesta en cuestión

\footnotetext{
1 Tesis de Maestría en Historia defendida el 11 de abril de 2019 en la Facultad de Humanidades de la Universidad Nacional de Mar de Plata, dirigida por la Dra. María Valeria Ciliberto (UNMdP / CONICET), y financiada mediante Beca B interna de la Universidad Nacional de Mar del Plata. El jurado evaluador estuvo compuesto por el Dr. Osvaldo Barreneche (UNLP / CONICET), la Dra. Gisela Sedeillán (UNMdP / CONICET) y el Dr. Pedro Berardi (UdeSA /CONICET). Calificación: 10 (diez) sobresaliente.

2 Proyecto de Investigación "Actores sociales, prácticas económicas y configuraciones institucionales (II): Buenos Aires ciudad y campaña (fines del siglo XVIII-mediados del siglo XIX)", Secretaría de Ciencia y Técnica, UNMdP. Radicado en el Grupo de Investigación "Problemas y Debates del siglo XIX" (G. XIX), CEHis, Facultad de Humanidades, UNMdP. Período: enero 2018-diciembre 2019. (HUM 575/18), Subsidio: 15/F637. ${ }^{3}$ Tesina de grado para alcanzar el grado de Licenciada. “Revolución, gobierno y orden social. La Intendencia de Policía de Buenos Aires (1812-1821)”, UNMdP, Defendida el 18 de mayo de 2015.
} 
de las historiografías oficiales, hechas por los propios comisarios con el objetivo de justificar el accionar policial y glorificar la institución.

El análisis que se desarrolla en esta tesis retoma los aportes de dicha renovación para abordar la configuración de las primeras formaciones policiales de la ciudad de Buenos Aires desde una mirada que abone a la construcción de una historia socio-política de la policía. La policía a la que aludimos a lo largo de la tesis es una que se debate entre función e institución, y muestra una combinación de elementos y nociones del Antiguo Régimen con las novedades institucionales que recorren el período propuesto, que incluye las Reformas Borbónicas, la década posterior a la Revolución de Mayo y el período de reformas impulsadas por el gobernador Martín Rodríguez y su ministro Bernardino Rivadavia.

El período que abordamos inicia con la conformación de las Intendencias a partir de la Real Ordenanza de Intendentes de 1782, sobre la cual se posan las primeras formaciones policiales, tales como la Intendencia de Policía que funciona desde 1799 hasta 1804 y luego la Intendencia de Policía establecida en 1812 por el Segundo Triunvirato. Ya contamos en este momento con las primeras divisiones territoriales de Buenos Aires que son acompañadas por figuras de control como los alcaldes de barrio, vecinos reconocidos que desarrollaban una tarea de vigilancia en tanto carga cívica/pública. Retomamos estas sucesivas reconfiguraciones institucionales, atendiendo aspectos normativos, recursos, actores y prácticas. Realizamos un corte en 1825, en primer lugar, porque condensa una serie de eventos políticos de importancia, como el reemplazo del gobernador Rodríguez por Las Heras, el incremento de las disidencias dentro de la elite durante el período constituyente de 1824-1826 (Ternavasio, 2004, p. 40) y el estallido del conflicto con el Brasil.

A su vez, la periodización está directamente relacionada con el objeto de estudio puesto que entre febrero y junio de 1825 se suprimen las comisarías de campaña, para luego ser reinstaladas, pero ahora con un reglamento propio, dando indicios de una primera separación entre el poder de policía que emanaba desde el casco urbano hasta aquel momento, y una policía específica para el ámbito rural. Esta cuestión es también el motivo de selección del espacio sobre el cual se desarrolla esta tesis, la ciudad de Buenos Aires y sus suburbios, en tanto en los años propuestos para el abordaje la policía es eminentemente urbana.

Hipotetizamos que la ejecución del poder policial pone en marcha prácticas policiales que muestran la combinación de viejos y nuevos modos de controlar, pero se asientan y valen de rasgos pertenecientes a un ordenamiento jurídico-político que pone el acento en la buena moral, en el individuo que la garantiza, en la confianza y virtud que inspira, en el conocimiento del terreno y la población a la que se vigila. Estos rasgos bien remiten a una sociedad que aún no ha desembarazado de su concepción corporativa. Las prácticas policiales, por ende, cristalizan esta yuxtaposición dando lugar a un poder de policía que avanza sobre el control poblacional, pero valiéndose de la confianza que generan sus miembros para concretarlo. 
La tesis se organiza en cuatro capítulos, precedidos por una introducción, y finaliza con una serie de reflexiones que dejan también preguntas abiertas y caminos a seguir e investigar en posteriores instancias de posgrado. Adjuntamos luego un apéndice documental que profundiza sobre las fuentes consultadas y la metodología utilizada. Privilegiamos un entrecruzamiento de fuentes, todas ellas de carácter oficial, y nos acercamos a las mismas desde una perspectiva cualitativa y ponderando una mirada relacionada con el estudio de caso. Nuestro acervo documental se compone por un corpus principal, compuesto por Órdenes Superiores, esto es, los partes diarios de la policía, que se encuentran contenidas en el Fondo Policía del Archivo General de la Nación, y por un corpus complementario, en donde combinamos documentos de tipo judicial (Juzgado del Crimen, Archivo Histórico de la Provincia de Buenos Aires) con otros éditos, como los Acuerdos del Cabildo, reglamentaciones y normativas contenidas en el Registro Oficial de la Provincia de Buenos Aires y censos, padrones y planos intervenidos.

El primer capítulo, titulado "Aproximaciones al objeto de estudio, estado de la cuestión y aportes teórico-metodológicos", condensa las líneas y áreas historiográficas que han permitido repensar un objeto y período sobre el cual ya disponemos de indagaciones destacadas (Barreneche, 2001; Casagrande, 2014), y colaborar con un examen que privilegia la construcción institucional del dispositivo policial integrando concepciones, normativas y prácticas. De este modo, hemos retomado elementos de la historia política renovada que posibilitaron volcar la mirada hacia "lo político" como acción y hacia la agencia de los sujetos intervinientes como parte de su construcción. Por su parte, la historia social de la justicia nos presentó los diálogos posibles entre la historia social, la historiografía rural rioplatense y la historia crítica del derecho, a los fines de abordar las tensiones entre justicia y policía y situarnos en un marco en donde las relaciones entre derecho, costumbre y ley no se presentan de forma lineal, complejizando los enfoques asentados en lo normativo. Los estudios policiales, por su parte, han sido clave en la ponderación de la interdisciplinariedad como constitutiva del campo en cuestión (Caimari y Sozzo, 2017, p. 10).

Los tres capítulos siguientes pasan al abordaje del objeto de estudio, interrelacionando tres ejes: espacio/territorio, normativa/recursos y actores/prácticas. A partir del análisis que se desarrolla allí es que sostenemos que las pervivencias en las concepciones y nociones jurídico-políticas de la policía, que la comprenden como parte del "buen gobierno" de la ciudad, son utilizadas y se combinan y yuxtaponen con innovaciones institucionales, que en forma conjunta pretenden dar respuesta a la necesidad de configurar un nuevo orden social luego de la Revolución.

El capítulo II, “La policía y el proceso de territorialización. Un proyecto de medio siglo", aborda, desde los conceptos de territorialidad y configuración del espacio político (Hespanha, 1993), los modos en que las primeras formaciones policiales emergen en conjunto con las divisiones territoriales que, iniciadas durante las Reformas Borbónicas, son retomadas por la dirigencia revolucionaria y post revolucionaria. Aquí los planos 
intervenidos que utilizamos ilustran el proceso de miniaturización del espacio a través de la instalación de cuarteles y a la vez, la ampliación del territorio controlado por la policía. Las divisiones territoriales se fisonomizan en tanto son equipadas con figuras específicas - primero, los alcaldes de barrio, luego también, los comisarios y celadoresque desarrollan tareas de vigilancia y control de la población y el territorio conjugándolas con otras funciones como la preocupación por la higiene y edificación urbana.

Los recursos humanos, normativos y materiales que colaboran con esta fisonomización son retomados en el capítulo III bajo el título “De la intendencia al Departamento: recursos, materialidad y funciones". Allí la periodización es ajustada y focaliza en los momentos en que se establece la Intendencia de Policía de 1812, luego reconfigurada en el Departamento de Policía en 1821, junto con la supresión de los Cabildos de Buenos Aires y Luján. El capítulo se divide en dos ejes, el primero, relativo a los reglamentos que acompañan a la Intendencia y al Departamento respectivamente y el segundo, interesado en observar el crecimiento material de la policía en términos de presupuesto, hombres, armas y animales. Se observa a través de estos años que la policía incrementa recursos institucionales de todo tipo como consecuencia de la ampliación de las funciones policiales que, a su vez, responde a una concepción de tipo paternalista doméstica de la policía (Casagrande, 2014, p. 121), lejana aún de cualquier especialización en la prevención del delito.

El capítulo IV, “Las prácticas policiales, un puente entre vigilados y vigilantes”, busca conocer quiénes eran los policías que efectivamente realizaban esas tareas y los modos en que llevaban adelante sus prácticas, interesándose por la construcción de la autoridad que se desenvolvía a través de ellas. De este modo, se posa la mirada, por un lado, sobre los intendentes y jefes y por otro, sobre los alcaldes de barrio. Mientras que los primeros compartían un perfil social que podemos considerar notable -eran militares, funcionarios, comerciantes-, la situación de los segundos se presenta más compleja en tanto sus perfiles se diferencian según el cuartel en el que deben cumplir funciones. Cerca del caso urbano y del poder político encontramos a alcaldes que se asemejan a los propios intendentes y jefes, mientras que, acercándonos a los suburbios y zona de quintas, quienes cumplen funciones policiales son labradores, horneros y panaderos. Esto sugiere que para emplazar una autoridad de tipo policial era necesario no solo conocer el terreno y la población a vigilar, sino también ser parte de ella a los fines de generar una confianza asentada en una proximidad que permita resolver conflictos de la manera más adecuada.

A lo largo de estos capítulos se pretende hilvanar tres ejes que consideramos constitutivos de estas primeras experiencias policiales en la ciudad de Buenos Aires y sus suburbios. La pervivencia de nociones y concepciones de policía ancladas en el Antiguo Régimen posibilita la construcción del dispositivo policial a través de diversas innovaciones, sean ellas de carácter normativo, material o espacial. El resultado es un proceso de configuración policial complejo que combina dichas innovaciones con un 
concepto de poder policial de tipo amplio y atado al buen gobierno de la ciudad. Esta combinatoria permite la efectivización de dicho poder de policía en el marco de la transición entre la colonia y el período independiente.

Los resultados parciales de esta investigación viabilizan la construcción de nuevos interrogantes, problemáticas y preguntas. Por caso, una ampliación temporal vehiculizaría una integración más profunda sobre los ensayos borbónicos de la policía, tanto en relación a los alcaldes de barrio como a la primera Intendencia de 1799 y sus conflictos jurisdiccionales con el Cabildo. A su vez, entre los actores intervinientes en la estructura policial es necesario abordar a los comisarios de ciudad y de campaña, cuyos perfiles y prácticas no pueden ser homologadas a las de los intendentes, jefes y alcaldes, sino que muestran aristas particulares. A su vez, esto es fundamental para ponderar el equipamiento político del territorio a través de las propias prácticas de la policía. La posibilidad de indagar sobre la relación entre prácticas y territorio y espacio político puede proveer una indagación con mayor detalle en torno al papel de la policía en el control sobre la circulación de información, mercancías y personas y su conexión con otros espacios institucionales en conformación. Estas posibles líneas de indagación son caminos a construir que esperamos retomar en el marco de la instancia doctoral.

\section{Referencias bibliográficas:}

Barreneche, O. (2001). Dentro de la ley, TODO. La justicia criminal de Buenos Aires en la etapa formativa del sistema penal moderno en Argentina. La Plata: Al Margen.

Casagrande, A. (2014). Vagos, Jueces y Policías: Una historia de la disciplina Económica en Buenos Aires (1785-1829). (Tesis de posgrado para optar por el grado de Doctor en Ciencias Sociales). Universidad Nacional de La Plata. La Plata. Disponible online en MemoriaAcadémica:

http://www.memoria.fahce.unlp.edu.ar/tesis/te.1046/te.1046.pdf.

Caimari, L. y Sozzo, M. (2017). Historia y cuestión criminal en América Latina: expansión, tendencias y desafíos. En L. Caimari y M. Sozzo (Eds.) Historia de la cuestión criminal en América Latina (pp. 9-25). Rosario: Prohistoria.

Hespanha, A. (1993). El espacio político. En La Gracia del Derecho. Economía de la cultura en la Edad Moderna (pp. 85-122). Madrid: Centro de Estudios Constitucionales.

Ternavasio, M. (2004). Construir poder y dividir poderes. Buenos Aires durante la "feliz experiencia" rivadaviana. Boletín del Instituto de Historia Argentina y Americana Dr. Emilio Ravignani 26, 7-43.

Para citar este resumen:

Vaccaroni, M. A. (2019). Resumen de tesis: “Policía y construcción estatal Higiene urbana, vigilancia y territorialidad en Buenos Aires y sus alrededores (1782-1824)". Anuario de la Escuela de Historia Virtual, 15, 91-95. 